\title{
Radiotherapy versus radiotherapy combined with temozolomide in high-risk low-grade gliomas after surgery: study protocol for a randomized controlled clinical trial
}

\author{
Jingjing Wang ${ }^{1+}$, Ying Wang ${ }^{2+}$, Yan He${ }^{1}$, Hui Guan ${ }^{1}$, Ling He${ }^{1}$, Xiaoli Mu ${ }^{1}$ and Xingchen Peng ${ }^{1 *}$ (I)
}

\begin{abstract}
Background: It has been reported that radiation therapy (RT) followed by procarbazine, lomustine, and vincristine (PCV) chemotherapy could improve progression-free survival (PFS) and overall survival (OS) in patients with high-risk World Health Organization (WHO) grade 2 gliomas after surgery. However, procarbazine is not available in China. In clinical practice, Chinese doctors often use radiotherapy combined with temozolomide (TMZ) to treat these patients, although large-scale prospective studies are lacking. This trial aims to confirm whether RT combined with temozolomide can improve PFS and OS in high-risk patients with low-grade gliomas (LGGs).

Methods/design: This is a two-group, randomized controlled trial (RCT) enrolling patients who have LGGs (WHO grade 2) and are aged 40 years or older without regard to the extent of resection or are aged younger than 40 years old with subtotal resection or biopsy. An estimated 250 patients will be enrolled. Eligible participants will be randomly assigned to receive RT alone or RT plus TMZ chemotherapy in a 1:1 ratio. The same RT will be given to all eligible participants regardless of whether they are randomly assigned to the RT group or the chemoradiotherapy (CRT) group. While in the CRT group, patients will receive adjuvant TMZ with or without concurrent radiochemotherapy. The primary outcome of this trial is PFS, and it will be analyzed by the intention-to-treat approach. Secondary outcomes include OS, adverse events, and cognitive function.
\end{abstract}

Discussion: The objective of our research is to assess the effect of radiotherapy coupled with TMZ in high-risk patients with LGGs after surgery, compared with RT alone. Different histological types and molecular subtypes will be examined, and a corresponding subgroup analysis will be conducted. Our data can provide evidence for postoperative adjuvant therapy in patients with high-risk LGGs in China.

Trial registration: Chinese Clinical Trial Registry, ChiCTR1800015199. Registered on 13 March 2018.

Keywords: Low-grade glioma, High-risk, Radiotherapy, Temozolomide

\footnotetext{
* Correspondence: pxx2014@scu.edu.cn

†ingjing Wang and Ying Wang contributed equally to this work.

${ }^{1}$ Department of Biotherapy, Cancer Center, West China Hospital, Sichuan

University, Chengdu 610041, Sichuan, China

Full list of author information is available at the end of the article
}

(c) The Author(s). 2019 Open Access This article is distributed under the terms of the Creative Commons Attribution 4.0 International License (http://creativecommons.org/licenses/by/4.0/) which permits unrestricted use, distribution, and reproduction in any medium, provided you give appropriate credit to the original author(s) and the source, provide a link to the Creative Commons license, and indicate if changes were made. The Creative Commons Public Domain Dedication waiver (http://creativecommons.org/publicdomain/zero/1.0/) applies to the data made available in this article, unless otherwise stated. 


\section{Background}

Grade 2 glioma accounts for about $15-20 \%$ of all brain tumors in adults [1]. According to the 2016 revision of the World Health Organization (WHO) classification of tumors of the central nervous system, the major pathological types of grade 2 low-grade gliomas (LGGs) include diffuse astrocytomas (wild-type IDH1, mutant $I D H 1$, or not otherwise specified) and oligodendrogliomas (mutant IDH1 and $1 \mathrm{p} / 19 \mathrm{q}$ co-deletion or not otherwise specified) [2]. The treatment modalities consist of surgery followed by observation, radiotherapy, chemotherapy, or chemoradiation [3]. Although surgery can cure a proportion of patients, some patients still have recurrence after surgery, and the LGGs can even transform into high-grade gliomas. Basing on longer survival data compared with high-grade gliomas, postoperative treatment decisions about observation versus aggressive treatments must take into account possible clinical benefits and side effects which may affect quality of life. Nowadays, postoperative adjuvant treatment strategies depend on whether the patient has high-risk factors. However, high-risk factors have been changing in recent years. Formerly, six factors were considered to be high-risk (astrocytoma, age $>40$ years, Karnofsky performance score $[\mathrm{KPS}]<70$, tumor dimension $>6 \mathrm{~cm}$, tumor crossing midline, preoperative neurological function deficits of moderate to severe degree). Since 2015, subtotal resection and age $>40$ years are regarded as main high-risk factors for LGGs [4]. Compared with the low-risk population, patients with high-risk factors need aggressive treatments after surgery. European Organisation for Research and Treatment of Cancer (EORTC) 22845 is a prospective trial to compare early radiotherapy with deferred radiotherapy [5]. A total of 314 patients with LGGs who had undergone surgical resection or biopsy were randomized to receive either early radiation therapy (RT) or delayed RT delivered when progression took place. The results showed that immediate postoperative radiotherapy improved progression-free survival (PFS) and provided better seizure control, but there was no impact on overall survival. Taken together, the results showed that patients with high-risk WHO grade 2 glioma can benefit from postoperative adjuvant RT. In addition, adjuvant RT alone and temozolomide (TMZ) alone were compared in EORTC 22033-26033 [6]. No differences in PFS were observed between the two arms. Furthermore, the question remains: Can radiotherapy combined with chemotherapy improve clinical outcomes in high-risk patients with LGG? Radiation Therapy Oncology Group (RTOG) 9802, a prospective clinical trial, indicated improvement in both PFS and overall survival (OS) with six cycles of adjuvant procarbazine, lomustine, and vincristine (PCV) chemotherapy following radiation, when compared with $\mathrm{RT}$ alone. In addition, the survival distribution continued to diverge over time $[7,8]$. However, procarbazine is not available in China. In clinical practice, Chinese doctors often use radiotherapy combined with TMZ to treat these patients, although large-scale prospective studies are lacking. TMZ is an alkylating agent with the chemical property to cross the blood-brain barrier. One standard treatment for high-grade glioma is to take TMZ as concurrent and adjuvant chemotherapy [9-11]. However, in LGGs, further studies are indispensable to ascertain the role of TMZ in addition to RT. RTOG 0424, a single-arm phase II study, combined concurrent and adjuvant TMZ with RT to treat patients with LGG with at least three risk factors for relapse (age $\geq 40$ years, preoperative tumor diameter $\geq$ $6 \mathrm{~cm}$, astrocytoma histology, tumor crossing the midline, or a preoperative neurological deficit of more than mild extent) [12]. The 3-year OS rate was $73.1 \%$, which significantly exceeded not only historical controls but also hypothesis. Although RTOG 0424 unveiled that radiotherapy followed by TMZ chemotherapy for high-risk LGGs had survival benefits, it was a single-arm trial, and Chinese data is still lacking. As a result, we decided to carry out a randomized controlled trial (RCT) to confirm the advantage of TMZ chemotherapy added to RT for patients with high-risk LGGs.

\section{Methods/design \\ Study objective}

The aim of this study is to compare the efficacy and safety of RT plus TMZ with RT alone for high-risk LGGs. Beyond that, we will focus on molecular features, in addition to histologic classification, to establish a more appropriate treatment modality for certain cohorts.

\section{Study design}

This is a multicenter, open-label, prospective RCT. The study has received ethical approval from the Chinese Ethics Committee of Registering Clinical Trials. The protocol is written in line with the Standard Protocol Items: Recommendations for Interventional Trials (SPIRIT) guidelines (see Additional file 1: SPIRIT checklist). Following informed consent, eligible patients will be allocated to an RT group (control group) or a chemoradiotherapy (CRT) group (experimental group). The flow diagram of the main procedures is illustrated in Fig. 1.

\section{Recruitment and informed consent}

A total of 250 participants will be recruited from four centers in China (see Additional file 2). Registration can 


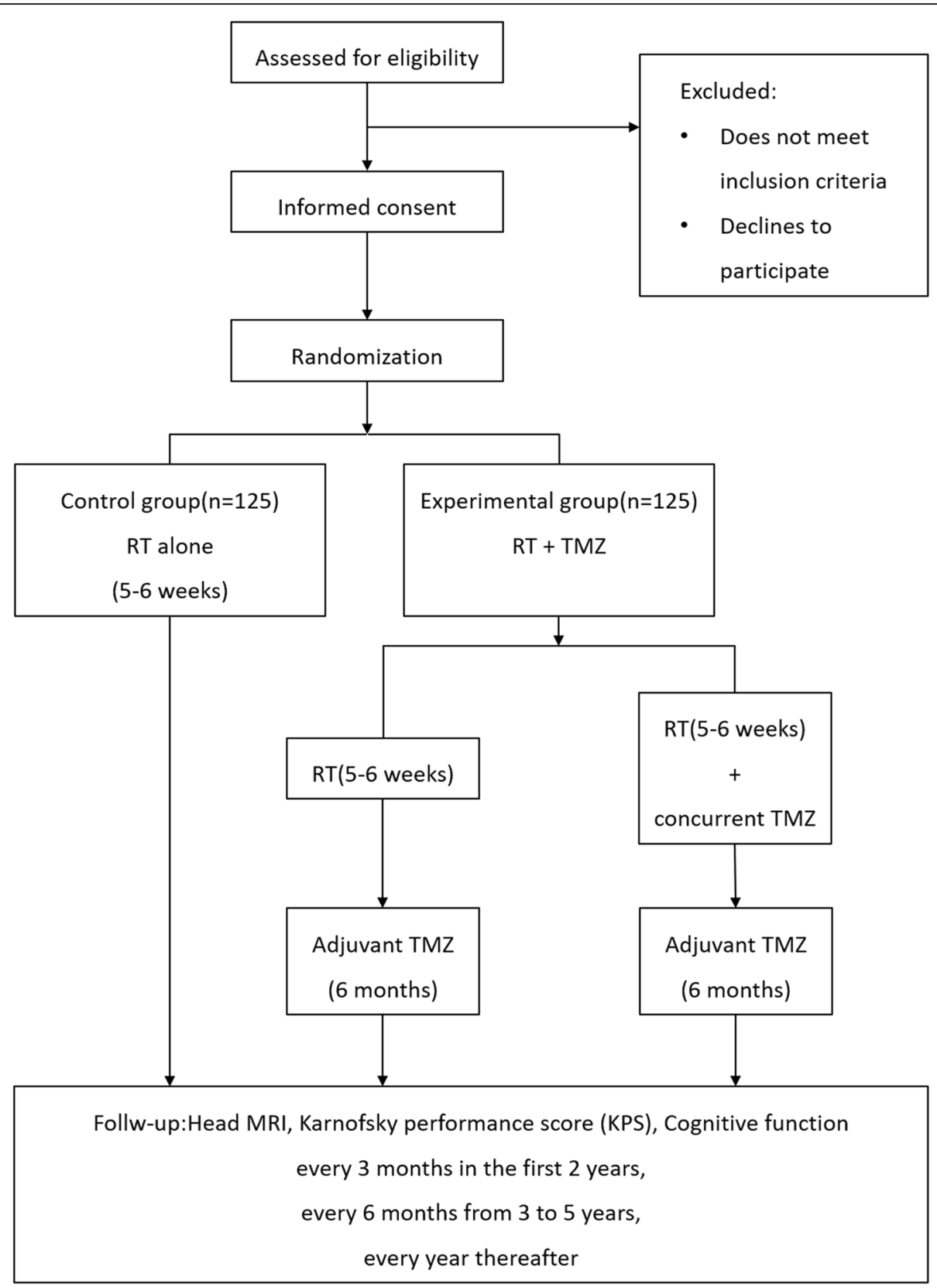

Fig. 1 Study flowchart. RT radiation therapy, TMZ temozolomide

be done at any time after a patient is diagnosed as having a WHO grade 2 glioma. The enrollment period is expected to be completed within 5 years from the beginning of recruitment. Research staff are in charge of the screening process to make sure each participant matches the inclusion and exclusion criteria. An informed consent form (ICF) describing the detailed study procedures and illustrating the potential benefits and risks will be provided to all participants, so that they can decide whether or not to volunteer. A signed written ICF must be acquired from all patients or their legal representatives prior to their participation in this clinical trial.

\section{Eligibility criteria}

The inclusion criteria are as follows:

1. Newly diagnosed supratentorial WHO grade 2 glioma

2. Aged 18-39 years without total resection, or aged 40-70 years with any extent of resection or biopsy

3. $\mathrm{KPS} \geq 60$ 
4. No more than moderate neurological symptoms or signs

5. The interval between surgery and randomization is less than 12 weeks

6. Have signed the ICF.

The exclusion criteria are as follows:

1. WHO grade 1 glioma or high-grade glioma according to WHO's grading system

2. Patient has received prior RT to the head and neck region

3. Patient has received prior chemotherapy

4. Synchronous multiple primary malignant tumors excluding carcinoma of the cervix in situ or nonmelanomatous skin cancer

5. Patient's prior malignancy disease-free survival less than 5 years

6. Patient has active infection

7. Patient is pregnant or breast-feeding.

\section{Randomization}

Qualified patients will be randomized in a 1:1 ratio using the method of block randomization. The blocked randomization sequence is generated by the SPSS computer software, and the block size is determined by statisticians. After the patient has registered and signed the ICF, he or she will be allocated to the RT group or the CRT group by web-based central randomization. The allocation sequence is unavailable to the researchers who are enrolling participants or assigning interventions.

\section{Interventions}

Eligible patients will be assigned 1:1 to the experimental (CRT) group or the control (RT) group. In the experimental group, patients will be treated with RT combined with adjuvant TMZ with or without concurrent TMZ. In the control group, patients will only have RT treatment. Both groups will receive the same intensitymodulated radiation therapy (IMRT). The radiation dose is $50-54$ Gy given in $25-30$ fractions $(1.8-2.0$ Gy once daily, 5 days per week). RT treatment volumes will be defined using preoperative and postoperative T2 or fluid-attenuated inversion recovery (FLAIR) on magnetic resonance imaging (MRI). Drug dose adjustments are allowed according to blood counts and adverse reactions. Concurrent chemotherapy is to receive oral TMZ, $75 \mathrm{mg} / \mathrm{m}^{2}$ per day, during RT. Concurrent TMZ can be used for 42 days continuously if the absolute neutrophil count (ANC) is not less than $1.5 \times 10^{9}$ cells per L, the platelet count is not less than $100 \times 10^{9}$ cells per $\mathrm{L}$, and there is a non-hematological toxicity of grade 0 or 1 (except for alopecia, nausea, and vomiting) according to version 4.03 of the National Cancer Institute Common
Toxicity Criteria (NCI-CTC). In the case of ANC less than $1.5 \times 10^{9}$ cells per $\mathrm{L}$ but more than $0.5 \times 10^{9}$ cells per L, platelet count less than $100 \times 10^{9}$ cells per L but more than $10 \times 10^{9}$ cells per L, or a non-hematological toxicity of grade 2 , concurrent TMZ treatment should suspend until recovery to toxicity of grade 0 or 1 . TMZ chemotherapy will be terminated when one of the following conditions occurs: ANC $<0.5 \times 10^{9}$ cells per $\mathrm{L}$, platelet count $<10 \times 10^{9}$ cells per $\mathrm{L}$, or non-hematological toxicity of grade 3 or 4 .

Patients who are assigned to adopt adjuvant chemotherapy will be treated with six cycles of TMZ, 150-200 $\mathrm{mg} / \mathrm{m}^{2}$ per day for 5 consecutive days, repeated every 4 weeks. There is a 28-day break during RT and adjuvant TMZ. The first cycle dose of adjuvant chemotherapy is $150 \mathrm{mg} / \mathrm{m}^{2}$. A higher dose of $200 \mathrm{mg} / \mathrm{m}^{2}$ is recommended in the subsequent cycles if ANC is not less than $1.5 \times 10^{9} / \mathrm{L}$, platelet count not less than $100 \times 10^{9} / \mathrm{L}$, and non-hematological toxicity of grade 0 or 1 (except for alopecia, nausea, and vomiting) during the first cycle. The dose will reduce by $50 \mathrm{mg} / \mathrm{m}^{2}$ if ANC is less than $1 \times 10^{9}$ cells per $\mathrm{L}$, platelet count is less than $50 \times 10^{9}$ cells per L, or there is non-hematological toxicity of grade 3 during any period of adjuvant chemotherapy. Patients must discontinue adjuvant TMZ if grade 4 nonhematological is recorded, the dose of TMZ has reduced to less than $100 \mathrm{mg} / \mathrm{m}^{2}$, or grade 3 non-hematological reoccurs after dose reduction.

\section{Outcomes}

Our primary outcome is PFS, which is calculated from the date of randomization to the date of first reported disease progression or the date of death from any cause. Secondary outcomes are OS, adverse events, and cognitive function. OS is calculated from the date of randomization until death from any cause. Cognitive function will be assessed by the Mini-Mental State Examination (MMSE).

\section{Adverse events (AEs)}

Investigators should explain to participants in detail that they are required to faithfully reflect changes in their condition during and after treatment. Researchers should pay close attention to AEs while observing the curative effect. The following information should be recorded in the case report form (CRF): symptom, occurrence time, severity, duration, treatment measures, and outcomes. Researchers should evaluate the association between $\mathrm{AE}$ and treatment and record them timely and truly with signature and date. The grading of AEs will be in accordance with CTC-AE version 4.03 . 


\section{Baseline and follow-up visits}

After obtaining written informed consent, a baseline assessment including a physical examination, KPS, complete blood count $(\mathrm{CBC})$, and serum biochemistry will be completed within 7 days before interventions. Postoperative head MRI and cognitive function test are allowed to complete within 28 days prior to treatment. During treatment, $\mathrm{CBC}$ and serum biochemistry (including renal and liver function, electrolytes, and lactate dehydrogenase) will be conducted weekly. The first follow-up visit (KPS, head MRI, cognitive function) will be 1 month after radiotherapy. These visits will then occur every 3 months during the first 2 years, every half year from 3 to 5 years, and at least annually thereafter. Participants will be followed up for 10 years after the end of treatment for the last patient. The schedule of assessments before, during, and after treatment is displayed in Table 1.

\section{Sample size}

This RCT is designed as a superiority trial. Based on literature reports and clinical experience, the median OS of the RT group is 7.8 years and that of the CRT group (RT plus TMZ) is 13.3 years. One-sided log-rank testing with a significance level of 0.05 , a test power of $80 \%$, and a withdrawal and loss of follow-up rate of $10 \%$ is applied in this trial. A 10-year follow-up for patient events will be conducted after the end of treatment for the last patient. The sample size estimated by PASS 11 software is 125 subjects per group.

\section{Data collection and management}

All relevant information for each subject should be recorded in the CRF and inputted in ResMan, an Internetbased electronic data capture system, timely and truly by trained research staff. The personal information of each subject is confidential. In order to promote participant retention, researchers will instruct subjects to take their medication as prescribed. Patients will also be informed of the follow-up visits by telephone in advance, and all items will be measured in strict accordance with the assessments schedule shown in Table 1. Two data entry staff are needed to input the data independently. After reviewing and confirming that the database is correct, electronic data will be conserved and backed up. As original material, the CRF is not easily changed. The researcher has to sign and date the CRF when it is necessary to modify. The locked electronic data files do not allow any changes to be made. The database will be statistically analyzed by statistical analysts as required by the statistical plan. The principal investigator has access to the final dataset, while other investigators are prohibited from entering. Except for the name-related data, the disclosure of the information to third parties is prohibited. After the completion of the trial, the responsible

Table 1 Study schedule of enrollment, interventions, and assessments

\begin{tabular}{|c|c|c|c|c|c|c|c|c|}
\hline \multirow[b]{3}{*}{ Time point } & \multicolumn{8}{|l|}{ Study period } \\
\hline & \multirow{2}{*}{$\begin{array}{l}\text { Enrollment } \\
-4 \text { weeks }\end{array}$} & \multirow{2}{*}{$\begin{array}{l}\text { Allocation } \\
0\end{array}$} & \multicolumn{6}{|c|}{ Post allocation } \\
\hline & & & Week 0-6 & Week 10 & Weeks 11-34 & Within 2 years & $3-5$ years & $5-10$ years \\
\hline \multicolumn{9}{|l|}{ Enrollment: } \\
\hline Eligibility screen & $x$ & & & & & & & \\
\hline Informed consent & $x$ & & & & & & & \\
\hline Randomization & $x$ & & & & & & & \\
\hline Allocation & & $x$ & & & & & & \\
\hline \multicolumn{9}{|l|}{ Interventions: } \\
\hline RT & & & $x$ & & & & & \\
\hline Concurrent TMZ & & & $x$ & & & & & \\
\hline Adjuvant TMZ & & & & $x$ & $x$ & & & \\
\hline \multicolumn{9}{|l|}{ Assessments: } \\
\hline Physical examination & & $x$ & & & & Every 3 months & Every 6 months & Annually \\
\hline KPS & & $x$ & & $x$ & & Every 3 months & Every 6 months & Annually \\
\hline $\mathrm{CBC}$ & & $x$ & Weekly & $x$ & Weekly & & & \\
\hline Serum biochemistry & & $x$ & Weekly & $x$ & Weekly & & & \\
\hline Postoperative head MRI & $x$ & & & $x$ & & Every 3 months & Every 6 months & Annually \\
\hline Cognitive function & $x$ & & & $x$ & & Every 3 months & Every 6 months & Annually \\
\hline Adverse events & & & $x$ & $x$ & $x$ & Every 3 months & Every 6 months & Annually \\
\hline
\end{tabular}

Abbreviations: $R T$ radiation therapy, TMZ temozolomide, KPS Karnofsky performance score, $C B C$ complete blood count, MRI magnetic resonance imaging 
unit of the study has the right to publish contents related to the experiment in the form of a paper.

\section{Data analysis}

Professional statisticians undertake statistical analysis tasks and participate in the whole process from trial design and implementation to analysis and summary. The Kaplan-Meier method will be used to estimate median PFS and OS, and a log-rank test will be used to compare differences between the two arms. Furthermore, a Cox proportional hazards analysis will be done to estimate the hazard ratio (HR) and 95\% confidence interval (CI). Regarding prognostic factors, univariate and multivariate analyses including age, histology, treatment method, $I D H$ mutation, $1 \mathrm{p} / 19 \mathrm{q}$ status, and $M G M T$ promoter status will be used to analyze their impact on PFS and OS. Safety analysis, mainly for AEs, will be done in the safety set (SS) population. All effectiveness analysis (PFS, OS, cognitive function) will be done on an intention-to-treat (ITT) set. The ITT analysis will be put to use to handle non-compliance and missing data.

\section{Data monitoring}

To ensure the safety and validity of the trial, the data will be overseen by an independent Data Safety Monitoring Board (DSMB) during the study period. The board consists of clinicians and statisticians and will monitor all implementation activities including but not limited to the enrollment of each center, starting time of procedures, and drop out. All AEs and issues concerning interventions will be reported to the DSMB in line with requirements. All data entered into the database will be checked by the DSMB before being locked, and no changes will be permitted. To ensure data security, data must be backed up in time, and irrelevant personnel cannot access and modify data.

\section{Discussion}

Optimal adjuvant management of adult low-grade gliomas (LGGs) is controversial. RTOG 9802 has shown striking survival improvements for patients with LGGs treated with adjuvant RT followed by PCV chemotherapy, and there is a significant average MMSE score increase in both arms [13]. But obviously, the incidence of AEs in the CRT group is higher than in the RT group. Thus, it is crucial to weigh the efficacy and safety of these treatments and further clarify how to combine them. Pathological molecular typing is an essential component of diagnosis and treatment of glioma at present. This clinical trial is the first large-scale prospective study to compare the effect of RT alone with RT plus TMZ involving molecular subtypes in high-risk patients with LGGs. The outcomes of this trial are expected to evaluate the predictive effects of diverse molecular markers
(IDH1/IDH2 mutations, 1p/19q co-deletion, MGMT promoter methylation status) and to find corresponding appropriate treatment patterns.

Cognitive function has aroused extensive concern in patients with brain tumors. EORTC 22033-26033, a prospective study of patients with LGGs, revealed no significant difference between the RT group and the TMZ group [14]. Therefore, it did not back the treatment of TMZ alone over RT alone. In our study, the MMSE, a widely used screening test for dementia and cognitive dysfunction and a practical approach for ranking the cognitive state [15-18], will be applied to assess cognitive function in both randomly assigned arms. It may affect the choice of individual therapeutic strategy for patients with LGGs if RT plus concomitant and adjuvant TMZ improves survival outcomes without additional cognitive function damage compared to RT alone.

\section{Trial status}

The final protocol version is 1.0, dated 11 February 2018. Patient recruitment began on 10 April 2018 after we acquired ethical approval, and it is ongoing. We anticipate the recruitment phase to be complete by April 2023.

\section{Supplementary information}

Supplementary information accompanies this paper at https://doi.org/10. 1186/s13063-019-3741-5.

Additional file 1. SPIRIT 2013 checklist: recommended items to address in a clinical trial protocol and related documents.

Additional file 2 Research centers.

\section{Abbreviations}

AE: Adverse event; ANC: Absolute neutrophil count; CBC: Complete blood count; Cl: Confidence interval; CRF: Case report form;

CRT: Chemoradiotherapy; DSMB: Data Safety Monitoring Board; FLAIR: Fluidattenuated inversion recovery; HR: Hazard ratio; ICF: Informed consent form; IDH: Isocitrate dehydrogenase; IMRT: Intensity-modulated radiation therapy; ITT: Intention-to-treat; KPS: Karnofsky performance score; LGG: Low-grade glioma; MMSE: Mini-Mental State Examination; MT: Mutant; NCI-CTC: National Cancer Institute Common Toxicity Criteria; OS: Overall survival;

PCV: Procarbazine, lomustine, and vincristine; PFS: Progression-free survival; RCT: Randomized controlled trial; RT: Radiation therapy, radiotherapy;

SPIRIT: Standard Protocol Items: Recommendations for Interventional Trials; SS: Safety set; TMZ: Temozolomide; WT: Wild-type

\section{Acknowledgements}

We express our sincere respect and heartfelt thanks to all of the patients for participating in this trial. The authors acknowledge support from all of the research nurses and staff and participating centers in this study.

\section{Authors' contributions}

$\mathrm{XCP}$ is the project designer and is responsible for the research. All authors participated in drafting the manuscript; XCP and $\mathrm{YW}$ revised it. XCP and $\mathrm{YH}$ are in charge of target volume delineation and the quality control of radiation. XCP, YW, YH, HG, and JJW participate in recruiting participants and obtaining informed consent, as well as assessing patients' cognitive function and filling out the MMSE form. JJW, YH, and HG are involved in data collection. LH and XLM contributed to performing the randomized sequence generation and will conduct the statistical analysis. XCP, JJW, YW, YH, and 
HG will monitor AEs and must report them in time. All authors have read and approved the final manuscript.

\section{Funding}

The study is supported by the National Natural Science Foundation of China (Grant Nos. 81672386 and 81402494) and West China Hospital, Sichuan University Science and Technology Commission (Grant No. H20182240254). The funding body will play no role in the design of the study, in collection, analysis, or interpretation of data, or in writing the manuscript.

\section{Availability of data and materials}

The datasets used and/or analyzed during the current study are available from the corresponding author on reasonable request.

\section{Ethics approval and consent to participate}

The trial protocol was registered on 13 March 2018 by the Chinese Clinical Trial Registry (ChiCTR1800015199). Central ethical approval has been confirmed from the Chinese Ethics Committee of Registering Clinical Trials (approval no. ChiECRCT-20180033) on 4 April 2018, and we will not begin recruiting at other centers in the trial until local ethical approval has been obtained. All participants have to provide a signed written ICF before enrollment.

\section{Consent for publication}

No personal identifying information will be published. Written informed consent must be acquired from all patients or their legal representatives for their clinical data to be published.

\section{Competing interests}

The authors declare that they have no competing interests.

\section{Author details}

${ }^{1}$ Department of Biotherapy, Cancer Center, West China Hospital, Sichuan University, Chengdu 610041, Sichuan, China. ${ }^{2}$ Geriatrics Center, West China Hospital, Sichuan University, Chengdu 610041, Sichuan, China.

Received: 25 March 2019 Accepted: 23 September 2019

Published online: 21 November 2019

\section{References}

1. Tandon A, Schiff D. Therapeutic decision making in patients with newly diagnosed low grade glioma. Curr Treat Options in Oncol. 2014;15(4): 529-38.

2. Komori T. The 2016 WHO Classification of Tumours of the Central Nervous System: the major points of revision. Neurol Med Chir. 2017;57(7):301-11.

3. Soffietti R, Baumert BG, Bello L, von Deimling A, Duffau H, Frenay M, Grisold W, Grant R, Graus F, Hoang-Xuan K, et al. Guidelines on management of low-grade gliomas: report of an EFNS-EANO Task Force. Eur J Neurol. 2010; 17(9):1124-33.

4. National Comprehensive Cancer Network (NCCN) Clinical Practice Guidelines in Oncology. Central Nervous System Cancers, Version 1.2015. https://www.nccn.org/professionals/physician_gls/default.aspx. Accessed 1 May 2015.

5. van den Bent MJ, Afra D, de Witte O, Ben Hassel M, Schraub S, Hoang-Xuan K, Malmstrom PO, Collette L, Pierart M, Mirimanoff R, et al. Long-term efficacy of early versus delayed radiotherapy for low-grade astrocytoma and oligodendroglioma in adults: the EORTC 22845 randomised trial. Lancet (London, England). 2005;366(9490):985-90.

6. Baumert BG, Hegi ME, van den Bent MJ, von Deimling A, Gorlia T, HoangXuan K, Brandes AA, Kantor G, Taphoorn MJ, Hassel MB, et al. Temozolomide chemotherapy versus radiotherapy in high-risk low-grade glioma (EORTC 22033-26033): a randomised, open-label, phase 3 intergroup study. Lancet Oncol. 2016;17(11):1521-32.

7. Shaw EG, Wang M, Coons SW, Brachman DG, Buckner JC, Stelzer KJ, Barger GR, Brown PD, Gilbert MR, Mehta MP. Randomized trial of radiation therapy plus procarbazine, lomustine, and vincristine chemotherapy for supratentorial adult low-grade glioma: initial results of RTOG 9802. J Clin Oncol. 2012;30(25):3065-70.

8. Buckner JC, Shaw EG, Pugh SL, Chakravarti A, Gilbert MR, Barger GR, Coons S, Ricci P, Bullard D, Brown PD, et al. Radiation plus procarbazine, CCNU, and vincristine in low-grade glioma. N Engl J Med. 2016;374(14):1344-55.
9. Stupp R, Mason WP, van den Bent MJ, Weller M, Fisher B, Taphoorn MJ, Belanger K, Brandes AA, Marosi C, Bogdahn U, et al. Radiotherapy plus concomitant and adjuvant temozolomide for glioblastoma. N Engl J Med. 2005:352(10):987-96

10. Hegi ME, Diserens AC, Gorlia T, Hamou MF, de Tribolet N, Weller M, Kros JM, Hainfellner JA, Mason W, Mariani L, et al. MGMT gene silencing and benefit from temozolomide in glioblastoma. N Engl J Med. 2005;352(10):997-1003.

11. van den Bent MJ, Baumert B, Erridge SC, Vogelbaum MA, Nowak AK, Sanson M, Brandes AA, Clement PM, Baurain JF, Mason WP, et al. Interim results from the CATNON trial (EORTC study 26053-22054) of treatment with concurrent and adjuvant temozolomide for $1 \mathrm{p} / 19 \mathrm{q}$ non-co-deleted anaplastic glioma: a phase 3, randomised, open-label intergroup study. Lancet (London, England). 2017;390(10103):1645-53.

12. Fisher BJ, Hu C, Macdonald DR, Lesser GJ, Coons SW, Brachman DG, Ryu S, Werner-Wasik M, Bahary JP, Liu J, et al. Phase 2 study of temozolomidebased chemoradiation therapy for high-risk low-grade gliomas: preliminary results of Radiation Therapy Oncology Group 0424. Int J Radiat Oncol Biol Phys. 2015;91(3):497-504

13. Prabhu RS, Won M, Shaw EG, Hu C, Brachman DG, Buckner JC, Stelzer KJ, Barger GR, Brown PD, Gilbert MR, et al. Effect of the addition of chemotherapy to radiotherapy on cognitive function in patients with low-grade glioma: secondary analysis of RTOG 98-02. J Clin Oncol. 2014; 32(6):535-41.

14. Reijneveld JC, Taphoorn MJ, Coens C, Bromberg JE, Mason WP, Hoang-Xuan K, Ryan G, Hassel MB, Enting RH, Brandes AA, et al. Health-related quality of life in patients with high-risk low-grade glioma (EORTC 22033-26033): a randomised, open-label, phase 3 intergroup study. Lancet Oncol. 2016; 17(11):1533-42.

15. Tombaugh TN, McIntyre NJ. The Mini-Mental State Examination: a comprehensive review. J Am Geriatr Soc. 1992;40(9):922-35.

16. Tangalos EG, Smith GE, Ivnik RJ, Petersen RC, Kokmen E, Kurland LT, Offord KP, Parisi JE. The Mini-Mental State Examination in general medical practice: clinical utility and acceptance. Mayo Clin Proc. 1996:71(9):829-37.

17. Salmon DP, Thal LJ, Butters N, Heindel WC. Longitudinal evaluation of dementia of the Alzheimer type: a comparison of 3 standardized mental status examinations. Neurology. 1990;40(8):1225-30.

18. Folstein MF, Folstein SE, McHugh PR. "Mini-mental state". A practical method for grading the cognitive state of patients for the clinician. J Psychiatr Res. 1975;12(3):189-98.

\section{Publisher's Note}

Springer Nature remains neutral with regard to jurisdictional claims in published maps and institutional affiliations.

Ready to submit your research? Choose BMC and benefit from:

- fast, convenient online submission

- thorough peer review by experienced researchers in your field

- rapid publication on acceptance

- support for research data, including large and complex data types

- gold Open Access which fosters wider collaboration and increased citations

- maximum visibility for your research: over $100 \mathrm{M}$ website views per year

At $\mathrm{BMC}$, research is always in progress.

Learn more biomedcentral.com/submissions 